\title{
Monopole Condensation and Polyakov Loop in Finite-Temperature Pure QCD
}

\author{
Shinji Ejiri a \\ ${ }^{\text {a }}$ Department of Physics, Kanazawa University, Kanazawa 920-11, Japan
}

\begin{abstract}
We study the relation between the abelian monopole condensation and the deconfinement phase transition of the finite-temperature pure QCD. The expectation value of the monopole contribution to the Polyakov loop becomes zero when a long monopole loop is distributed uniformly in the configuration of the confinement phase. On the other hand, it becomes non-zero when the long monopole loop disappears in the deconfinement phase. We also discuss the relation between the monopole behaviors and the usual interpretation of the spontaneous breaking of $\mathrm{Z}(\mathrm{N})$ symmetry in finite-temperature $\mathrm{SU}(\mathrm{N}) \mathrm{QCD}$. It is found that the boundary condition of the space direction is important to understand the $\mathrm{Z}(\mathrm{N})$ symmetry in terms of the monopoles.
\end{abstract}

\section{Introduction}

The purpose of this study is to confirm if the quark confinement mechanism in QCD can be understood as dual Meissner effect due to abelian monopole condensation. This idea is supported by recent results of Monte-Carlo simulations of abelian projected QCD. The remarkable results in finite-temperature QCD is the followings: (1) The configuration of the monopole currents changes dramatically at $T_{c}$ when we adopt the maximally abelian gauge. (2) The monopole contribution to the Polyakov loop shows similar behavior to the original Polyakov loop and the residual photon part of the Polyakov loop is finite even in the confinement phase. It means that the monopole part controls the deconfinement transition [1]. (3) Last year, we found that the behavior of the Polyakov loop can be explained by the monopole dynamics, considering the 3-dimensional solid angle in $\mathrm{SU}(2)$ gauge theory [2].

Let us explain briefly the interpretation using the 3-dimensional solid angle. First, updation of link variables is done as usual. Then, we perform abelian projection 13. gauge field.

$$
\begin{aligned}
U_{\mu}^{\prime}(s) & =C_{\mu}(s) u_{\mu}(s), \\
u_{\mu} & =\operatorname{diag}\left(e^{i \theta_{\mu}^{(1)}}, \cdots, e^{i \theta_{\mu}^{(N)}}\right), \quad \operatorname{det} u_{\mu}=1,
\end{aligned}
$$

where $U_{\mu}^{\prime}$ is the gauge fixed link field, $u_{\mu}$ is the abelian link field, and $\theta_{\mu}^{(i)}$ can be regarded as the abelian gauge field. We adopt the maximally abelian gauge. The monopole current $k_{\mu}(s)$ is defined as

$$
\begin{aligned}
k_{\mu}(s) & =(1 / 4 \pi) \epsilon_{\mu \alpha \beta \gamma} \partial_{\alpha} \bar{\Theta}_{\beta \gamma}(s), \\
\Theta_{\mu \nu}(s) & =\partial_{\mu} \theta_{\nu}(s)-\partial_{\nu} \theta_{\mu}(s)=\bar{\Theta}_{\mu \nu}(s)+2 \pi n_{\mu \nu}(s),
\end{aligned}
$$

where $\bar{\Theta}_{\mu \nu} \in[-\pi, \pi)$ and $n_{\mu \nu}$ is an integer, following DeGrand-Toussaint [5]. Here, ${ }^{*} n_{\mu \nu}(s)=$ $\frac{1}{2} \epsilon_{\mu \nu \rho \sigma} n_{\rho \sigma}(s)$ is the Dirac string: $k_{\mu}=\partial_{\nu}{ }^{*} n_{\nu \mu}$. The abelian Polyakov loop operator $P_{a}$ and the contributions from the monopole $P_{m}$ and the photon $P_{p}$ are defined as follows [1]:

$$
\begin{aligned}
P_{a} & =\frac{1}{N} \sum_{i} \exp \left\{i \sum_{j=0}^{N_{t}-1} \theta_{4}^{(i)}(s+j \hat{4})\right\}=P_{p} \cdot P_{m},(3) \\
P_{p} & =\frac{1}{N} \sum_{i} \exp \left\{-i \sum_{j, s^{\prime}} D\left(s+j \hat{4}-s^{\prime}\right) \partial_{\nu}^{\prime} \bar{\Theta}_{\nu 4}^{(i)}\left(s^{\prime}\right)\right\},(4) \\
P_{m} & =\frac{1}{N} \sum_{i} \exp \left\{-2 \pi i \sum_{j, s^{\prime}} D\left(s+j \hat{4}-s^{\prime}\right) \partial_{\nu}^{\prime} n_{\nu 4}^{(i)}\left(s^{\prime}\right)\right\},
\end{aligned}
$$

where $D\left(s-s^{\prime}\right)$ is the lattice Coulomb propagator satisfying: $\partial_{\mu}^{\prime} \partial_{\mu} D(s)=-\delta_{s, 0}$.

Here we restrict ourselves to the monopole part. If we integrate over the time direction, the monopole part of the Polyakov loop can be rewritten in the infinite-volume limit as follows:

$P_{m}(\vec{s})=\exp \left(2 \pi i \frac{\Omega(\vec{s})}{4 \pi}\right)$, 
where $\Omega(\vec{s})$ is the oriented solid angle made by monopole loops looking from Polyakov loop $(\vec{s})$ in the 3-dimensional reduced space. The properties of Polyakov loop can be understood by the monopole dynamics qualitatively.

In the confinement phase $\left(\mathrm{T}<\mathrm{T}_{\mathrm{c}}\right)$, long monopole loops distribute uniformly [6]. The $\Omega$ can be random from 0 to $4 \pi$. The Polyakov loop at each point is random. The average of the Polyakov loop is zero. On the other hand, in the deconfinement phase $\left(\mathrm{T}>\mathrm{T}_{\mathrm{c}}\right)$, there is a space where no monopole exists. In such a space, $\Omega$ takes small value, and the local Polyakov loops are nearly one. Hence, the average of the Polyakov loop approaches one. This picture is confirmed by the measurement of the histogram of the solid angle using Monte-Carlo simulations. However, the effect of the boundary condition in the finite-volume was not considered in that explanation. Here we study the relation between the monopole dynamics and the $\mathrm{Z}(\mathrm{N})$ symmetry that controls the behavior of the Polyakov loop in $\mathrm{SU}(2)$ and $\mathrm{SU}(3)$ gauge theory. We found that the boundary condition in the finite volume analysis is very important to understand the $\mathrm{Z}(\mathrm{N})$ symmetry in terms of the monopole.

\section{Boundary Conditions}

When the periodic boundary condition is adopted, the lattice Coulomb propagator can not be defined exactly. We use the following approximation for the Coulomb propagator:

$\partial_{\mu}^{\prime} \partial_{\mu} D(s)=-\delta_{s, 0}+\frac{1}{N_{\text {site }}}$,

where $N_{\text {site }}$ is the number of sites. Notice that there exist many closed Dirac sheets (Bubbles of Dirac sheet). A bubble of Dirac sheet does not contribute to the Polyakov loop when the Coulomb propagator is exact. However, in the periodic boundary condition, the bubble of Dirac sheet gives the finite phase of the monopole part of the abelian Polyakov loop due to the incompleteness of the propagator:

$P_{m}=\exp \left\{2 \pi i \frac{N_{b}}{N_{\text {space }}}\right\}$, where $N_{\text {space }}$ and $N_{b}$ are the number of sites and the number of sites inside a bubble in 3dimensional space respectively. It is found that the case corresponding to the $\mathrm{Z}(\mathrm{N})$ phase, i.e., $N_{b} / N_{\text {space }}=k / N(k$ : integer $)$ alone keeps the action invariant and then is important.

Next, let us consider the anti-periodic boundary condition (C-periodic boundary condition). The lattice Coulomb propagator can be defined exactly by imposing the anti-periodic boundary condition for the space direction:

$D\left(s+N_{s} \hat{i}\right)=-D(s), \quad \partial_{\mu}^{\prime} \partial_{\mu} D(s)=-\delta_{s, 0}$.

Then the gauge fields and the monopole currents must satisfy the anti-periodic boundary condition:

$\theta_{\mu}\left(s+N_{s} \hat{i}\right)=-\theta_{\mu}(s), k_{\mu}\left(s+N_{s} \hat{i}\right)=-k_{\mu}(s)(10)$

and the link fields must obey C-periodic boundary condition [7]:

$U_{\mu}\left(s+N_{s} \hat{i}\right)=U_{\mu}^{*}(s) \quad$ (complex conjugate).

The solid angle interpretation in the infinitevolume limit is applicable also in this boundary condition. But the global Z(3) symmetry is broken explicitly in the case of $\mathrm{SU}(3)$.

\section{3. $\mathrm{SU}(2)$ case}

The histogram of the Polyakov loop (averaged over $\vec{x}$ ) in the deconfinement phase of the $\mathrm{SU}(2)$ gauge theory is shown in Fig.1. The histogram of the Polyakov loop are similar in both boundary conditions, but the interpretation in terms of monopoles are quite different. In the case of the periodic boundary condition, the $\mathrm{Z}(2)$ symmetry is given by the contribution from the bubble of Dirac sheet due to the incompleteness of the Coulomb propagator. On the other hand, in the case of the anti-periodic (C-periodic) boundary condition, there is a degree of freedom of introducing infinite size of Dirac sheet without changing the action. The infinite size of Dirac sheet gives the $\mathrm{Z}(2)$ symmetry. It gives $\Omega=2 \pi$. If we add this Dirac string, $\left\langle P_{m}\right\rangle \rightarrow-\left\langle P_{m}\right\rangle$. The action does not change by the transformation putting the infinite size of Dirac sheet on every time slice. 

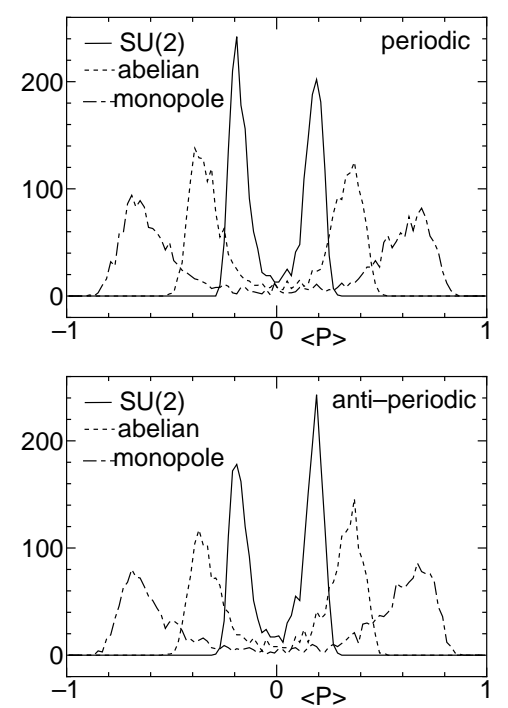

Figure 1. The histogram of the Polyakov loop (averaged over $\vec{x}$ ), the abelian Polyakov loop and the monopole part in the $\mathrm{SU}(2)$ gauge theory with periodic and anti-periodic boundary conditions. $\left(12^{3} \times 4\right.$ lattice, $\left.\beta=2.32\right)$

\section{4. $\mathrm{SU}(3)$ case}

Fig. 2 is the distribution of the Polyakov loop (averaged over $\vec{x}$ ) in the $\mathrm{SU}(3)$ gauge theory. In the periodic boundary condition, the global Z(3) symmetry is exact. But the lattice Coulomb propagator can not be defined exactly. The effect of the bubble of Dirac sheet due to the incompleteness of the propagator gives the $\mathrm{Z}(3)$ phases as in $\mathrm{SU}(2)$ case. The number of monopoles on each phase of the Polyakov loop is about the same in the deconfinement phase. On the other hand, in the anti-periodic (C-periodic) boundary condition, the solid angle explanation can be applicable, since the lattice Coulomb propagator is exact. The $\mathrm{Z}(3)$ symmetry is broken by the boundary condition. In the deconfinement phase, the Polyakov loop are distributed only in the vicinity of the real axis, This distribution can be understood by the monopole dynamics using the solid angle expression.

The author thanks T.Suzuki, Y.Matsubara, V.Bornyakov, S.Kitahara and F.Shoji for useful
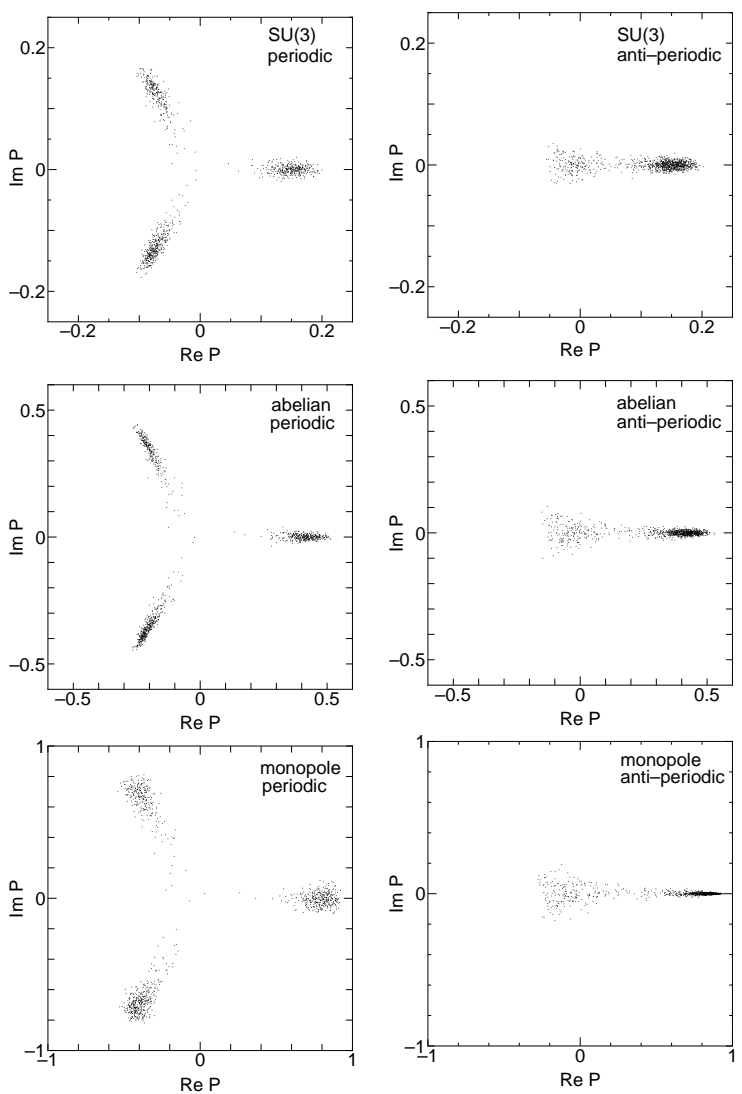

Figure 2. The distribution of the Polyakov loop (averaged over $\vec{x}$ ), the abelian Polyakov loop and the monopole part in the $\mathrm{SU}(3)$ gauge theory with periodic and anti-periodic boundary conditions. $\left(12^{3} \times 4\right.$ lattice, $\left.\beta=5.71\right)$

discussions and comments.

\section{REFERENCES}

1. T. Suzuki et al., Phys. Lett. B347 (1995) 375.

2. S. Ejiri et al., Nucl. Phys. B(Proc. Suppl.) 47 (1996) 322.

3. G. 'tHooft, Nucl. Phys. B190 (1981) 455.

4. A.S. Kronfeld et al., Phys. Lett. B198 (1987) 516; A.S.Kronfeld et al., Nucl.Phys. B293 (1987) 461.

5. T.A. DeGrand and D. Toussaint, Phys. Rev. D22 (1980) 2478.

6. S. Kitahara, Y. Matsubara and T. Suzuki, Prog. Theor. Phys. 93 (1995) 1. 
7. A.S. Kronfeld and U.-J. Wiese, Nucl. Phys. B357 (1991) 521; A.S. Kronfeld and U.-J. Wiese, Nucl. Phys. B401 (1993) 190. 